\title{
Strong convergence of a splitting algorithm for treating monotone operators
}

\author{
Sun Young Cho' ${ }^{1}$ Xiaolong Qin ${ }^{2,3}$ and Lin Wang ${ }^{4 *}$
}

\section{"Correspondence:}

wl64mail@aliyun.com

${ }^{4}$ College of Statistics and

Mathematics, Yunnan University of

Finance and Economics, Kunming,

650221, China

Full list of author information is

available at the end of the article

\begin{abstract}
In this paper, we investigate a splitting algorithm for treating monotone operators. Strong convergence theorems are established in the framework of Hilbert spaces.

Keywords: maximal monotone operator; fixed point; nonexpansive mapping; proximal point algorithm; zero point
\end{abstract}

\section{Introduction and preliminaries}

In this article, we always assume that $H$ is a real Hilbert space with inner product $\langle\cdot, \cdot\rangle$ and norm $\|\cdot\|$ and $C$ is a nonempty, closed, and convex subset of $H$.

Let $S: C \rightarrow C$ be a mapping. $F(S)$ stands for the fixed point set of $S . S$ is said to be contractive iff there exists a constant $\alpha \in(0,1)$ such that

$$
\|S x-S y\| \leq \alpha\|x-y\|, \quad \forall x, y \in C .
$$

It is well known that every contractive mapping has a unique fixed point in metric spaces. $S$ is said to be nonexpansive iff

$$
\|S x-S y\| \leq\|x-y\|, \quad \forall x, y \in C .
$$

If $C$ is a bounded, closed, and convex subset of $H$, then $F(S)$ is not empty, closed, and convex; see [1] and the references therein. $S$ is said to be strictly pseudocontractive iff there exists a constant $\kappa \in[0,1)$ such that

$$
\|S x-S y\|^{2} \leq\|x-y\|^{2}+\kappa\|x-y-S x+S y\|^{2}, \quad \forall x, y \in C .
$$

The class of strictly pseudocontractive mapping was introduced by Browder and Petryshyn [2]. It is clear that the class of strictly pseudocontractive mappings include the class of nonexpansive mappings as a special case. It is also not hard to see that strictly pseudocontractive mapping is continuous.

Let $A: C \rightarrow H$ be a mapping. Recall that $A$ is said to be monotone iff

$$
\langle A x-A y, x-y\rangle \geq 0, \quad \forall x, y \in C .
$$

O2014 Cho et al.; licensee Springer. This is an Open Access article distributed under the terms of the Creative Commons Attribution License (http://creativecommons.org/licenses/by/2.0), which permits unrestricted use, distribution, and reproduction in any medium, provided the original work is properly cited. 
$A$ is said to be strongly monotone iff there exists a constant $\kappa>0$ such that

$$
\langle A x-A y, x-y\rangle \geq \kappa\|x-y\|^{2}, \quad \forall x, y \in C .
$$

$A$ is said to be inverse-strongly monotone iff there exists a constant $\kappa>0$ such that

$$
\langle A x-A y, x-y\rangle \geq \kappa\|A x-A y\|^{2}, \quad \forall x, y \in C .
$$

$A$ is inverse-strongly monotone iff the inverse of $A$ is strongly monotone. It is not hard to see that every inverse-strongly monotone mapping is monotone and continuous. Let $I$ be the identity mapping on $H$. From [2], we know that $I-S$ is inverse-strongly monotone iff $S$ is strictly pseudocontractive; for more details, see [2] and the references therein.

The classical variational inequality problem is formulated as finding a point $x \in C$ such that

$$
\langle y-x, A x\rangle \geq 0, \quad \forall y \in C
$$

Such a point $x \in C$ is called a solution of the variational inequality. In this paper, we use $V I(C, A)$ to denote the solution set of the variational inequality. It is known that $x$ is a solution of the variational inequality iff $x$ is a fixed point of the mapping $\operatorname{Proj}_{C}(I-r A)$, where $\operatorname{Proj}_{C}$ is the metric projection from $H$ onto $C, I$ is the identity and $r$ is some positive real number. Recently, many authors studied solutions of inverse-strongly monotone variational inequalities based on the equivalence; see [3-13].

Recall that a set-valued mapping $B: H \rightrightarrows H$ is said to be monotone iff, for all $x, y \in H$, $f \in B x$ and $g \in B y$ imply $\langle x-y, f-g\rangle>0$. In this paper, we use $B^{-1}(0)$ to stand for the zero point of $B$. A monotone mapping $B: H \rightrightarrows H$ is maximal iff the graph $\operatorname{Graph}(B)$ of $B$ is not properly contained in the graph of any other monotone mapping. It is known that a monotone mapping $B$ is maximal if and only if, for any $(x, f) \in H \times H,\langle x-y, f-g\rangle \geq 0$, for all $(y, g) \in \operatorname{Graph}(B)$ implies $f \in B x$. For a maximal monotone operator $B$ on $H$, and $r>0$, we may define the single-valued resolvent $J_{r}: H \rightarrow \operatorname{Dom}(B)$, where $\operatorname{Dom}(B)$ denote the domain of $B$. It is known that $J_{r}$ is firmly nonexpansive, and $B^{-1}(0)=F\left(J_{r}\right)$.

One of the most important techniques for solving zero point problem of monotone operators goes back to the work of Browder [14]. Many important problems have reformulations which require finding zero points, for instance, evolution equations, complementarity problems, mini-max problems, variational inequalities and fixed point problems. It is well known that minimizing a convex function $f$ can be reduced to finding zero points of the subdifferential mapping $A=\partial f$. One of the basic ideas in the case of a Hilbert space $H$ is reducing the above inclusion problem to a fixed point problem of the operator $R_{A}$ defined by $R_{A}=(I+A)^{-1}$, which is called the classical resolvent of $A$. If $A$ has some monotonicity conditions, the classical resolvent of $A$ is with full domain and firmly nonexpansive. The property of the resolvent ensures that the Picard iterative algorithm $x_{n+1}=R_{A} x_{n}$ converge weakly to a fixed point of $R_{A}$, which is necessarily a zero point of $A$. Rockafellar introduced this iteration method and call it the proximal point algorithm (PPA); for more details, see [15] and [16] and the references therein.

It is known that PPA is only convergent and it was also pointed in [17] that it is often impractical since, in many cases, to solve the fixed point problem exactly is either impossible 
or of the same difficult as the original zero point problem. Therefore, one of the most interesting and important problems in the theory of monotone operators is to find an efficient iterative algorithm to compute their zero points. In many disciplines, including economics [18], image recovery [19], quantum physics [20], and control theory [21], problems arises in infinite dimension spaces. In such problems, strong convergence (norm convergence) is often much more desirable than weak convergence, for it translates the physically tangible property that the energy $\left\|x_{n}-x\right\|$ of the error between the iterate $x_{n}$ and the solution $x$ eventually becomes arbitrarily small. The important of strong convergence is also underlined in [22], where a convex function $f$ is minimized via the proximal point algorithm: it is shown that the rate of convergence of the value sequence $\left\{f\left(x_{n}\right)\right\}$ is better when $\left\{x_{n}\right\}$ converges strongly that it converges weakly. Such properties have a direct impact when the process is executed directly in the underlying infinite dimensional space.

To improve the weak convergence of PPA, many authors considered lots of different modifications; see [23-36] the references therein. One of the classic results was established by Solodov and Svaiter [33]. They obtained strong convergence theorems in Hilbert space without any compact assumption but with the aid of the metric projection.

In this paper, we are concerned with the problem of finding an element in the zero point set of the sum of two operators which are inverse-strongly monotone and a maximal monotone and in the fixed point set of a mapping which is strictly pseudocontractive. Strong convergence theorems are established without the aid of the metric projections. The organization of this paper is as follows. In Section 1, we provide an introduction and some necessary preliminaries. In Section 2, a regularization iterative algorithm is investigated. A strong convergence theorem is established without the aid of metric projections. In Section 3, applications of the main results are discussed.

In order to prove our main results, we also need the following lemmas.

Lemma 1.1 [36] Let $A: C \rightarrow H$ be a mapping, and $B: H \rightrightarrows H$ a maximal monotone operator. Then $F\left(J_{r}(I-r B)\right)=(A+B)^{-1}(0)$.

Lemma 1.2 [37] Let $E$ be a Banach space and let $A$ be an m-accretive operator. For $\lambda>0$, $\mu>0$, and $x \in E$, we have $J_{\lambda} x=J_{\mu}\left(\frac{\mu}{\lambda} x+\left(1-\frac{\mu}{\lambda}\right) J_{\lambda} x\right)$, where $J_{\lambda}=(I+\lambda A)^{-1}$ and $J_{\mu}=(I+\mu A)^{-1}$.

Lemma 1.3 [38] Let $\left\{x_{n}\right\}$ and $\left\{y_{n}\right\}$ be bounded sequences in a Banach space E, and $\left\{\beta_{n}\right\}$ be a sequence in $(0,1)$ with $0<\liminf _{n \rightarrow \infty} \beta_{n} \leq \limsup _{n \rightarrow \infty} \beta_{n}<1$. Suppose that $x_{n+1}=$ $\left(1-\beta_{n}\right) y_{n}+\beta_{n} x_{n}, \forall n \geq 1$ and

$$
\limsup _{n \rightarrow \infty}\left(\left\|y_{n+1}-y_{n}\right\|-\left\|x_{n+1}-x_{n}\right\|\right) \leq 0 .
$$

Then $\lim _{n \rightarrow \infty}\left\|y_{n}-x_{n}\right\|=0$.

Lemma 1.4 [39] Let $\left\{a_{n}\right\}$ be a sequence of nonnegative numbers satisfying the condition $a_{n+1} \leq\left(1-t_{n}\right) a_{n}+t_{n} b_{n}+c_{n}, \forall n \geq 0$, where $\left\{t_{n}\right\}$ is a number sequence in $(0,1)$ such that $\lim _{n \rightarrow \infty} t_{n}=0$ and $\sum_{n=0}^{\infty} t_{n}=\infty,\left\{b_{n}\right\}$ is a number sequence such that $\lim _{\sup _{n \rightarrow \infty}} b_{n} \leq 0$, and $\left\{c_{n}\right\}$ is a positive number sequence such that $\sum_{n=0}^{\infty} c_{n}<\infty$. Then $\lim _{n \rightarrow \infty} a_{n}=0$.

Lemma 1.5 [40] Let $S: C \rightarrow C$ be a strictly pseudocontractive mapping with the constant $\kappa \in[0,1)$. Then $S$ is Lipschitz continuous and $I-S$ is demiclosed at zero. Define a mapping 
$T: C \rightarrow C$ by $T x:=a x+(1-a)$ Sx for each $x \in C$. Then, as $a \in[\kappa, 1), T$ is nonexpansive such that $F(S)=F(T)$.

\section{Convergence analysis}

Theorem 2.1 Let $A: C \rightarrow H$ be an $\alpha$-inverse-strongly monotone mapping and let $B$ be a maximal monotone operator on $H$. Let $S: C \rightarrow C$ be a strictly pseudocontractive mapping with the constant $\kappa \in[0,1)$ and let $f: C \rightarrow C$ be a contractive mapping with the constant $\beta \in[0,1)$. Assume that $\operatorname{Dom}(B) \subset C$ and $F(S) \cap(A+B)^{-1}(0)$ is not empty. Let $J_{r_{n}}=\left(I+r_{n} B\right)^{-1}$ and let $\left\{x_{n}\right\}$ be a sequence generated in the following process: $x_{0} \in C$ and

$$
\left\{\begin{array}{l}
z_{n}=\alpha_{n} f\left(x_{n}\right)+\left(1-\alpha_{n}\right) x_{n}, \\
y_{n}=J_{r_{n}}\left(z_{n}-r_{n} A z_{n}+e_{n}\right), \\
x_{n+1}=\beta_{n} x_{n}+\left(1-\beta_{n}\right)\left(\gamma_{n} y_{n}+\left(1-\gamma_{n}\right) S y_{n}\right), \quad \forall n \geq 0,
\end{array}\right.
$$

where $\left\{\alpha_{n}\right\},\left\{\beta_{n}\right\}$ and $\left\{\gamma_{n}\right\}$ are real number sequences in $(0,1)$ and $\left\{r_{n}\right\}$ is a positive real number sequence in $(0,2 \alpha)$. Assume that the control sequences satisfy the following restrictions:

(a) $\lim _{n \rightarrow \infty} \alpha_{n}=0, \sum_{n=0}^{\infty} \alpha_{n}=\infty$;

(b) $0<\liminf _{n \rightarrow \infty} \beta_{n} \leq \limsup _{n \rightarrow \infty} \beta_{n}<1$;

(c) $\kappa \leq \gamma_{n} \leq a<1, \lim _{n \rightarrow \infty}\left|\gamma_{n+1}-\gamma_{n}\right|=0$;

(d) $0<b \leq r_{n} \leq c<2 \alpha$ and $\sum_{n=1}^{\infty}\left|r_{n}-r_{n-1}\right|<\infty$;

(e) $\sum_{n=0}^{\infty}\left\|e_{n}\right\|<\infty$,

where $a, b$ and $c$ are three real numbers. Then $\left\{x_{n}\right\}$ converges strongly to a point $\bar{x} \in F(S) \cap$ $(A+B)^{-1}(0)$, where $\bar{x}=\operatorname{Proj}_{F(S) \cap(A+B)^{-1}(0)} f(\bar{x})$.

Proof First, we show that $\left\{x_{n}\right\}$ is bounded. Notice that $I-r_{n} A$ is nonexpansive. Indeed, we have

$$
\begin{aligned}
\|( & \left(-r_{n} A\right) x-\left(I-r_{n} A\right) y \|^{2} \\
& =\|x-y\|^{2}-2 r_{n}\langle x-y, A x-A y\rangle+r_{n}{ }^{2}\|A x-A y\|^{2} \\
& \leq\|x-y\|^{2}-r_{n}\left(2 \alpha-r_{n}\right)\|A x-A y\|^{2} .
\end{aligned}
$$

In view of the restriction (d), we find that $I-r_{n} A$ is nonexpansive. Fixing $p \in F(S) \cap(A+$ $B)^{-1}(0)$, we find that

$$
\begin{aligned}
\left\|z_{n}-p\right\| & \leq \alpha_{n}\left\|f\left(x_{n}\right)-p\right\|+\left(1-\alpha_{n}\right)\left\|x_{n}-p\right\| \\
& \leq\left(1-\alpha_{n}(1-\beta)\right)\left\|x_{n}-p\right\|+\alpha_{n}\|f(p)-p\| .
\end{aligned}
$$

Putting $T_{n} x:=\gamma_{n} x+\left(1-\gamma_{n}\right) S x$ for each $x \in C$, we see from Lemma 1.5 that $T_{n}$ is nonexpansive with $F\left(T_{n}\right)=F(S)$ for each $n \geq 0$. It follows that

$$
\begin{aligned}
\left\|x_{n+1}-p\right\| & \leq \beta_{n}\left\|x_{n}-p\right\|+\left(1-\beta_{n}\right)\left\|T_{n} J_{r_{n}}\left(z_{n}-r_{n} A z_{n}+e_{n}\right)-p\right\| \\
& \leq \beta_{n}\left\|x_{n}-p\right\|+\left(1-\beta_{n}\right)\left\|z_{n}-p\right\|+\left(1-\beta_{n}\right)\left\|e_{n}\right\| \\
& \leq \beta_{n}\left\|x_{n}-p\right\|+\left(1-\alpha_{n}(1-\beta)\right)\left(1-\beta_{n}\right)\left\|x_{n}-p\right\|
\end{aligned}
$$




$$
\begin{aligned}
& +\alpha_{n}\left(1-\beta_{n}\right)\|f(p)-p\|+\left\|e_{n}\right\| \\
\leq & \left(1-\alpha_{n}(1-\beta)\left(1-\beta_{n}\right)\right)\left\|x_{n}-p\right\|+\alpha_{n}\left(1-\beta_{n}\right)\|f(p)-p\|+\left\|e_{n}\right\| \\
\leq & \max \left\{\left\|x_{n}-p\right\|, \frac{\|f(p)-p\|}{1-\beta}\right\}+\left\|e_{n}\right\| \\
\leq & \max \left\{\left\|x_{n-1}-p\right\|, \frac{\|f(p)-p\|}{1-\beta}\right\}+\left\|e_{n-1}\right\|+\left\|e_{n}\right\| \\
& \vdots \\
\leq & \max \left\{\left\|x_{0}-p\right\|, \frac{\|f(p)-p\|}{1-\beta}\right\}+\sum_{i=0}^{n}\left\|e_{i}\right\| \\
\leq & \max \left\{\left\|x_{0}-p\right\|, \frac{\|f(p)-p\|}{1-\beta}\right\}+\sum_{i=0}^{\infty}\left\|e_{i}\right\|<\infty .
\end{aligned}
$$

This proves that the sequence $\left\{x_{n}\right\}$ is bounded, so are $\left\{y_{n}\right\}$ and $\left\{z_{n}\right\}$. Notice that

$$
\left\|z_{n}-z_{n-1}\right\| \leq\left(1-\alpha_{n}(1-\beta)\right)\left\|x_{n}-x_{n-1}\right\|+\left|\alpha_{n}-\alpha_{n-1}\right|\left\|f\left(x_{n-1}\right)-x_{n-1}\right\| .
$$

Putting $\rho_{n}=z_{n}-r_{n} A z_{n}+e_{n}$, we find that

$$
\begin{aligned}
\left\|\rho_{n}-\rho_{n-1}\right\| \leq & \left\|z_{n}-z_{n-1}\right\|+\left\|r_{n}-r_{n-1}\right\|\left\|A z_{n-1}\right\|+\left\|e_{n}\right\|+\left\|e_{n-1}\right\| \\
\leq & \left(1-\alpha_{n}(1-\beta)\right)\left\|x_{n}-x_{n-1}\right\|+\left|\alpha_{n}-\alpha_{n-1}\right|\left\|f\left(x_{n-1}\right)-x_{n-1}\right\| \\
& \quad+\left|r_{n}-r_{n-1}\right|\left\|A z_{n-1}\right\|+\left\|e_{n}\right\|+\left\|e_{n-1}\right\| .
\end{aligned}
$$

It follows from Lemma 1.2 that

$$
\begin{aligned}
\left\|y_{n}-y_{n-1}\right\| & =\left\|J_{r_{n-1}}\left(\frac{r_{n-1}}{r_{n}} \rho_{n}+\left(1-\frac{r_{n-1}}{r_{n}}\right) J_{r_{n}} \rho_{n}\right)-J_{r_{n-1}} \rho_{n-1}\right\| \\
& \leq\left\|\frac{r_{n-1}}{r_{n}}\left(\rho_{n}-\rho_{n-1}\right)+\left(1-\frac{r_{n-1}}{r_{n}}\right)\left(J_{r_{n}} \rho_{n}-\rho_{n-1}\right)\right\| \\
& \leq\left\|\left(\rho_{n}-\rho_{n-1}\right)+\left(1-\frac{r_{n-1}}{r_{n}}\right)\left(J_{r_{n}} \rho_{n}-\rho_{n}\right)\right\| \\
& \leq\left\|\rho_{n}-\rho_{n-1}\right\|+\frac{\left|r_{n}-r_{n-1}\right|}{b}\left\|J_{r_{n}} \rho_{n}-\rho_{n}\right\| \\
& \leq\left(1-\alpha_{n}(1-\beta)\right)\left\|x_{n}-x_{n-1}\right\|+f_{n} \\
& \leq\left\|x_{n}-x_{n-1}\right\|+f_{n},
\end{aligned}
$$

where

$$
f_{n}=\left|\alpha_{n}-\alpha_{n-1}\right|\left\|f\left(x_{n-1}\right)-x_{n-1}\right\|+\left|r_{n}-r_{n-1}\right|\left(\left\|A z_{n-1}\right\|+\frac{\left\|J_{r_{n}} \rho_{n}-\rho_{n}\right\|}{b}\right)+\left\|e_{n}\right\|+\left\|e_{n-1}\right\| .
$$

This implies that

$$
\begin{aligned}
\left\|T_{n} y_{n}-T_{n-1} y_{n-1}\right\| & \leq\left\|T_{n} y_{n}-T_{n} y_{n-1}\right\|+\left\|T_{n} y_{n-1}-T_{n-1} y_{n-1}\right\| \\
& \leq\left\|x_{n}-x_{n-1}\right\|+f_{n}+\left|\gamma_{n}-\gamma_{n-1}\right|\left\|S y_{n-1}-y_{n-1}\right\| .
\end{aligned}
$$


In view of the restrictions (a), (c), (d), and (e), we find that

$$
\limsup _{n \rightarrow \infty}\left(\left\|T_{n} y_{n}-T_{n-1} y_{n-1}\right\|-\left\|x_{n}-x_{n-1}\right\|\right) \leq 0 .
$$

It follows from Lemma 1.3 that

$$
\lim _{n \rightarrow \infty}\left\|T_{n} y_{n}-x_{n}\right\|=0
$$

This in turn implies that

$$
\lim _{n \rightarrow \infty}\left\|x_{n+1}-x_{n}\right\|=0
$$

Notice that

$$
\begin{aligned}
\left\|x_{n+1}-p\right\|^{2} \leq & \beta_{n}\left\|x_{n}-p\right\|^{2}+\left(1-\beta_{n}\right)\left\|T_{n} J_{r_{n}} \rho_{n}-p\right\|^{2} \\
\leq & \beta_{n}\left\|x_{n}-p\right\|^{2}+\left(1-\beta_{n}\right)\left\|J_{r_{n}}\left(z_{n}-r_{n} A z_{n}+e_{n}\right)-p\right\|^{2} \\
\leq & \beta_{n}\left\|x_{n}-p\right\|^{2}+\left(1-\beta_{n}\right)\left\|\left(z_{n}-r_{n} A z_{n}\right)-\left(I-r_{n} A\right) p\right\|^{2} \\
& \quad+\left\|e_{n}\right\|\left(\left\|e_{n}\right\|+2\left\|\left(z_{n}-r_{n} A z_{n}\right)-\left(I-r_{n} A\right) p\right\|\right) \\
\leq & \beta_{n}\left\|x_{n}-p\right\|^{2}+\left(1-\beta_{n}\right)\left\|z_{n}-p\right\|^{2}-r_{n}\left(1-\beta_{n}\right)\left(2 \alpha-r_{n}\right)\left\|A z_{n}-A p\right\|^{2} \\
& \quad+\left\|e_{n}\right\|\left(\left\|e_{n}\right\|+2\left\|\left(z_{n}-r_{n} A z_{n}\right)-\left(I-r_{n} A\right) p\right\|\right) \\
\leq & \beta_{n}\left\|x_{n}-p\right\|^{2}+\alpha_{n}\left(1-\beta_{n}\right)\left\|f\left(x_{n}\right)-p\right\|^{2}+\left(1-\beta_{n}\right)\left(1-\alpha_{n}\right)\left\|x_{n}-p\right\|^{2} \\
& \quad-r_{n}\left(1-\beta_{n}\right)\left(2 \alpha-r_{n}\right)\left\|A z_{n}-A p\right\|^{2}+g_{n},
\end{aligned}
$$

where $g_{n}=\left\|e_{n}\right\|\left(\left\|e_{n}\right\|+2\left\|\left(z_{n}-r_{n} A z_{n}\right)-\left(I-r_{n} A\right) p\right\|\right)$. It follows that

$$
\begin{aligned}
& r_{n}\left(1-\beta_{n}\right)\left(2 \alpha-r_{n}\right)\left\|A z_{n}-A p\right\|^{2} \\
& \quad \leq\left\|x_{n}-p\right\|^{2}-\left\|x_{n+1}-p\right\|^{2}+\alpha_{n}\left(1-\beta_{n}\right)\left\|f\left(x_{n}\right)-p\right\|^{2}+g_{n} \\
& \quad \leq\left(\left\|x_{n}-p\right\|+\left\|x_{n+1}-p\right\|\right)\left\|x_{n+1}-x_{n}\right\|+\alpha_{n}\left\|f\left(x_{n}\right)-p\right\|^{2}+g_{n} .
\end{aligned}
$$

In view of the restrictions (a), (b), (c), (d), and (e), we find from (2.2) that

$$
\lim _{n \rightarrow \infty}\left\|A z_{n}-A p\right\|=0
$$

Since $J_{r_{n}}$ is firmly nonexpansive, we find that

$$
\begin{aligned}
\left\|J_{r_{n}} \rho_{n}-p\right\|^{2} \leq & \left\langle J_{r_{n}} \rho_{n}-p,\left(z_{n}-r_{n} A z_{n}+e_{n}\right)-\left(p-r_{n} A p\right)\right\rangle \\
= & \frac{1}{2}\left(\left\|J_{r_{n}} \rho_{n}-p\right\|^{2}+\left\|\left(z_{n}-r_{n} A z_{n}+e_{n}\right)-\left(p-r_{n} A p\right)\right\|^{2}\right. \\
& \left.-\left\|\left(J_{r_{n}} \rho_{n}-p\right)-\left(\left(z_{n}-r_{n} A z_{n}+e_{n}\right)-\left(p-r_{n} A p\right)\right)\right\|^{2}\right) \\
\leq & \frac{1}{2}\left(\left\|J_{r_{n}} \rho_{n}-p\right\|^{2}+\left\|\left(I-r_{n} A\right) z_{n}-\left(I-r_{n} A\right) p\right\|^{2}+g_{n}\right.
\end{aligned}
$$




$$
\begin{aligned}
& \left.-\left\|J_{r_{n}} \rho_{n}-z_{n}-e_{n}+r_{n} A z_{n}-r_{n} A p\right\|^{2}\right) \\
\leq & \frac{1}{2}\left(\left\|J_{r_{n}} \rho_{n}-p\right\|^{2}+\left\|z_{n}-p\right\|^{2}+g_{n}-\left\|J_{r_{n}} \rho_{n}-z_{n}-e_{n}\right\|^{2}\right. \\
& \left.-\left\|r_{n} A z_{n}-r_{n} A p\right\|^{2}+2 r_{n}\left\|A z_{n}-A p\right\|\left\|J_{r_{n}} \rho_{n}-z_{n}-e_{n}\right\|\right) .
\end{aligned}
$$

It follows that

$$
\begin{aligned}
\left\|J_{r_{n}} \rho_{n}-p\right\|^{2} \leq & \left\|z_{n}-p\right\|^{2}+g_{n}-\left\|J_{r_{n}} \rho_{n}-z_{n}-e_{n}\right\|^{2} \\
& -\left\|r_{n} A z_{n}-r_{n} A p\right\|^{2}+2\left\|e_{n}\right\|\left\|J_{r_{n}} \rho_{n}-z_{n}+r_{n} A z_{n}-r_{n} A p\right\| \\
\leq & \alpha_{n}\left\|f\left(x_{n}\right)-p\right\|^{2}+\left(1-\alpha_{n}\right)\left\|x_{n}-p\right\|^{2}+g_{n}-\left\|J_{r_{n}} \rho_{n}-z_{n}-e_{n}\right\|^{2} \\
& +2 r_{n}\left\|A z_{n}-A p\right\|\left\|J_{r_{n}} \rho_{n}-z_{n}-e_{n}\right\| .
\end{aligned}
$$

This implies that

$$
\begin{aligned}
\left\|x_{n+1}-p\right\|^{2} \leq & \beta_{n}\left\|x_{n}-p\right\|^{2}+\left(1-\beta_{n}\right)\left\|T_{n} J_{r_{n}} \rho_{n}-p\right\|^{2} \\
\leq & \beta_{n}\left\|x_{n}-p\right\|^{2}+\left(1-\beta_{n}\right)\left\|J_{r_{n}} \rho_{n}-p\right\|^{2} \\
\leq & \left\|x_{n}-p\right\|^{2}+\alpha_{n}\left\|f\left(x_{n}\right)-p\right\|^{2}+g_{n}-\left(1-\beta_{n}\right)\left\|J_{r_{n}} \rho_{n}-z_{n}-e_{n}\right\|^{2} \\
& +2 r_{n}\left\|A z_{n}-A p\right\|\left\|J_{r_{n}} \rho_{n}-z_{n}-e_{n}\right\| .
\end{aligned}
$$

It follows that

$$
\begin{aligned}
\left(1-\beta_{n}\right)\left\|J_{r_{n}} \rho_{n}-z_{n}-e_{n}\right\|^{2} \leq & \left(\left\|x_{n}-p\right\|+\left\|x_{n+1}-p\right\|\right)\left\|x_{n}-x_{n+1}\right\|+\alpha_{n}\left\|f\left(x_{n}\right)-p\right\|^{2} \\
& +g_{n}+2 r_{n}\left\|A z_{n}-A p\right\|\left\|J_{r_{n}} \rho_{n}-z_{n}-e_{n}\right\| .
\end{aligned}
$$

In view of the restrictions (a), (b), and (e), we find from (2.2) and (2.3) that $\lim _{n \rightarrow \infty} \| J_{r_{n}} \rho_{n}-$ $z_{n}-e_{n} \|=0$. This in turn implies that

$$
\lim _{n \rightarrow \infty}\left\|J_{r_{n}} \rho_{n}-z_{n}\right\|=0 .
$$

Notice that

$$
\begin{aligned}
\left\|y_{n}-x_{n}\right\| & \leq\left\|y_{n}-z_{n}\right\|+\left\|z_{n}-x_{n}\right\| \\
& \leq\left\|y_{n}-z_{n}\right\|+\alpha_{n}\left\|f\left(x_{n}\right)-x_{n}\right\| .
\end{aligned}
$$

It follows from (2.4) that

$$
\lim _{n \rightarrow \infty}\left\|y_{n}-x_{n}\right\|=0
$$

On the other hand, we have

$$
\begin{aligned}
\left\|T_{n} x_{n}-x_{n}\right\| \leq & \left\|\left(\gamma_{n} x_{n}+\left(1-\gamma_{n}\right) S x_{n}\right)-\left(\gamma_{n} y_{n}+\left(1-\gamma_{n}\right) S y_{n}\right)\right\| \\
& +\left\|\left(\gamma_{n} y_{n}+\left(1-\gamma_{n}\right) S y_{n}\right)-x_{n}\right\| \\
\leq & \gamma_{n}\left\|y_{n}-x_{n}\right\|+\left(1-\gamma_{n}\right)\left\|S y_{n}-S x_{n}\right\|+\left\|T_{n} y_{n}-x_{n}\right\| .
\end{aligned}
$$


Since $S$ is Lipschitz continuous, we find from (2.1) and (2.5) that

$$
\lim _{n \rightarrow \infty}\left\|T_{n} x_{n}-x_{n}\right\|=0
$$

Notice that

$$
\begin{aligned}
\left\|S y_{n}-y_{n}\right\| & \leq\left\|S y_{n}-T_{n} y_{n}\right\|+\left\|T_{n} y_{n}-T_{n} x_{n}\right\|+\left\|T_{n} x_{n}-x_{n}\right\|+\left\|x_{n}-y_{n}\right\| \\
& \leq \gamma_{n}\left\|y_{n}-S y_{n}\right\|+2\left\|y_{n}-x_{n}\right\|+\left\|T_{n} x_{n}-x_{n}\right\| .
\end{aligned}
$$

That is,

$$
\left(1-\gamma_{n}\right)\left\|S y_{n}-y_{n}\right\| \leq 2\left\|y_{n}-x_{n}\right\|+\left\|T_{n} x_{n}-x_{n}\right\|
$$

In view of (2.5) and (2.6), we find from the restriction (c) that

$$
\lim _{n \rightarrow \infty}\left\|S y_{n}-y_{n}\right\|=0
$$

Since $\operatorname{Proj}_{F(S) \cap(A+B)^{-1}(0)} f$ is contractive, we see that there exists a unique fixed point, say $\bar{x}$. Next, we show that $\limsup _{n \rightarrow \infty}\left\langle f(\bar{x})-\bar{x}, z_{n}-\bar{x}\right\rangle \leq 0$. To show it, we can choose a subsequence $\left\{z_{n_{i}}\right\}$ of $\left\{z_{n}\right\}$ such that

$$
\limsup _{n \rightarrow \infty}\left\langle f(\bar{x})-\bar{x}, z_{n}-\bar{x}\right\rangle=\lim _{i \rightarrow \infty}\left\langle f(\bar{x})-\bar{x}, z_{n_{i}}-\bar{x}\right\rangle
$$

Since $\left\{z_{n_{i}}\right\}$ is bounded, we can choose a subsequence $\left\{z_{n_{i}}\right\}$ of $\left\{z_{n_{i}}\right\}$ which converges weakly to some point $x$. We may assume, without loss of generality, that $z_{n_{i}}$ converges weakly to $x$. In view of (2.4), we find that $y_{n_{i}}$ also converges weakly to $x$. It follows from Lemma 1.5 that $x \in F(S)$.

Now, we are in a position to show that $x \in(A+B)^{-1}(0)$. Notice that $y_{n}=J_{r_{n}}\left(z_{n}-r_{n} A z_{n}+\right.$ $\left.e_{n}\right)$. It follows that

$$
z_{n}-r_{n} A z_{n}+e_{n} \in\left(I+r_{n} B\right) y_{n}
$$

That is,

$$
\frac{z_{n}-y_{n}}{r_{n}}-A z_{n}+e_{n} \in B y_{n}
$$

Since $B$ is monotone, we get, for any $(\mu, \nu) \in B$,

$$
\left\langle y_{n}-\mu, \frac{z_{n}-y_{n}}{r_{n}}-A z_{n}+e_{n}-v\right\rangle \geq 0 .
$$

Replacing $n$ by $n_{i}$ and letting $i \rightarrow \infty$, we obtain from (2.4) that

$$
\langle x-\mu,-A x-v\rangle \geq 0 \text {. }
$$


This gives $-A x \in B x$, that is, $0 \in(A+B)(x)$. This proves that $x \in(A+B)^{-1}(0)$. This complete the proof that $x \in F(S) \cap(A+B)^{-1}(0)$. It follows that

$$
\limsup _{n \rightarrow \infty}\left\langle f(\bar{x})-\bar{x}, z_{n}-\bar{x}\right\rangle \leq 0
$$

Finally, we show that $x_{n} \rightarrow \bar{x}$. Notice that

$$
\begin{aligned}
\left\|z_{n}-\bar{x}\right\|^{2} \leq & \alpha_{n}\left\langle f\left(x_{n}\right)-\bar{x}, z_{n}-\bar{x}\right\rangle+\left(1-\alpha_{n}\right)\left\|x_{n}-\bar{x}\right\|\left\|z_{n}-\bar{x}\right\| \\
\leq & \alpha_{n}\left\|f\left(x_{n}\right)-f(\bar{x})\right\|\left\|z_{n}-\bar{x}\right\|+\alpha_{n}\left\langle f(\bar{x})-\bar{x}, z_{n}-\bar{x}\right\rangle \\
& +\left(1-\alpha_{n}\right)\left\|x_{n}-\bar{x}\right\|\left\|z_{n}-\bar{x}\right\| \\
\leq & \frac{1-\alpha_{n}(1-\beta)}{2}\left(\left\|x_{n}-\bar{x}\right\|^{2}+\left\|z_{n}-\bar{x}\right\|^{2}\right)+\alpha_{n}\left\langle f(\bar{x})-\bar{x}, z_{n}-\bar{x}\right\rangle .
\end{aligned}
$$

This implies that

$$
\left\|z_{n}-\bar{x}\right\|^{2} \leq\left(1-\alpha_{n}(1-\beta)\right)\left\|x_{n}-\bar{x}\right\|^{2}+2 \alpha_{n}\left\langle f(\bar{x})-\bar{x}, z_{n}-\bar{x}\right\rangle .
$$

It follows that

$$
\begin{aligned}
\left\|y_{n}-\bar{x}\right\|^{2} \leq & \left\|\left(z_{n}-r_{n} A z_{n}\right)-\left(\bar{x}-r_{n} A \bar{x}\right)+e_{n}\right\|^{2} \\
\leq & \left\|\left(z_{n}-r_{n} A z_{n}\right)-\left(\bar{x}-r_{n} A \bar{x}\right)\right\|^{2}+\left\|e_{n}\right\|^{2} \\
& +2\left\|e_{n}\right\|\left\|\left(z_{n}-r_{n} A z_{n}\right)-\left(\bar{x}-r_{n} A \bar{x}\right)\right\| \\
\leq & \left\|z_{n}-\bar{x}\right\|^{2}+h_{n} \\
\leq & \left(1-\alpha_{n}(1-\beta)\right)\left\|x_{n}-\bar{x}\right\|^{2}+2 \alpha_{n}\left\langle f(\bar{x})-\bar{x}, z_{n}-\bar{x}\right\rangle+h_{n},
\end{aligned}
$$

where $h_{n}=\left\|e_{n}\right\|\left(\left\|e_{n}\right\|+2\left\|\left(z_{n}-r_{n} A z_{n}\right)-\left(I-r_{n} A\right) \bar{x}\right\|\right)$. It follows from (2.8) that

$$
\begin{aligned}
\left\|x_{n+1}-\bar{x}\right\|^{2} \leq & \beta_{n}\left\|x_{n}-\bar{x}\right\|^{2}+\left(1-\beta_{n}\right)\left\|T_{n} y_{n}-\bar{x}\right\|^{2} \\
\leq & \beta_{n}\left\|x_{n}-\bar{x}\right\|^{2}+\left(1-\beta_{n}\right)\left\|y_{n}-\bar{x}\right\|^{2} \\
\leq & \left(1-\alpha_{n}\left(1-\beta_{n}\right)(1-\beta)\right)\left\|x_{n}-\bar{x}\right\|^{2} \\
& +2 \alpha_{n}\left(1-\beta_{n}\right)\left(f(\bar{x})-\bar{x}, z_{n}-\bar{x}\right\rangle+h_{n} .
\end{aligned}
$$

In view of the restriction (a), (b), and (e), we find from Lemma 1.4 that $x_{n} \rightarrow \bar{x}$. This completes the proof.

If $S$ is nonexpansive and $\gamma_{n} \equiv 0$, then we have the following result immediately.

Corollary 2.2 Let $A: C \rightarrow H$ be an $\alpha$-inverse-strongly monotone mapping and let $B$ be a maximal monotone operator on $H$. Let $S: C \rightarrow C$ be a nonexpansive mapping and let $f: C \rightarrow C$ be a contractive mapping with the constant $\beta \in[0,1)$. Assume that $\operatorname{Dom}(B) \subset C$ and $F(S) \cap(A+B)^{-1}(0)$ is not empty. Let $J_{r_{n}}=\left(I+r_{n} B\right)^{-1}$ and let $\left\{x_{n}\right\}$ be a sequence generated 
in the following process: $x_{0} \in C$ and

$$
\left\{\begin{array}{l}
y_{n}=\alpha_{n} f\left(x_{n}\right)+\left(1-\alpha_{n}\right) x_{n}, \\
x_{n+1}=\beta_{n} x_{n}+\left(1-\beta_{n}\right) S J_{r_{n}}\left(y_{n}-r_{n} A y_{n}+e_{n}\right), \quad \forall n \geq 0,
\end{array}\right.
$$

where $\left\{\alpha_{n}\right\}$ and $\left\{\beta_{n}\right\}$ are real number sequences in $(0,1)$ and $\left\{r_{n}\right\}$ is a positive real number sequence in $(0,2 \alpha)$. Assume that the control sequences satisfy the following restrictions:

(a) $\lim _{n \rightarrow \infty} \alpha_{n}=0, \sum_{n=0}^{\infty} \alpha_{n}=\infty$;

(b) $0<\liminf _{n \rightarrow \infty} \beta_{n} \leq \limsup _{n \rightarrow \infty} \beta_{n}<1$;

(c) $0<b \leq r_{n} \leq c<2 \alpha$ and $\sum_{n=1}^{\infty}\left|r_{n}-r_{n-1}\right|<\infty$;

(d) $\sum_{n=0}^{\infty}\left\|e_{n}\right\|<\infty$,

where $b$ and c are two real numbers. Then $\left\{x_{n}\right\}$ converges strongly to a point $\bar{x} \in F(S) \cap(A+$ $B)^{-1}(0)$, where $\bar{x}=\operatorname{Proj}_{F(S) \cap(A+B)^{-1}(0)} f(\bar{x})$.

\section{Applications}

Many nonlinear problems arising in applied areas such as image recovery, signal processing, and machine learning are mathematically modeled as a nonlinear operator equation and this operator is decomposed as the sum of two nonlinear operators. The central problem is to iteratively find a zero point of the sum of two monotone operators, that is,

$$
0 \in(A+B)(x)
$$

Many real word problems can be formulated as a problem of the above form. For instance, a stationary solution to the initial value problem of the evolution equation

$$
\left\{\begin{array}{l}
0 \in F u+\frac{\partial u}{\partial t} \\
u_{0}=u(0)
\end{array}\right.
$$

can be recast as the inclusion problem when the governing maximal monotone $F$ is of the form $F=A+B$; for more details, see [41] and the references therein.

First, we give the following result.

Theorem 3.1 Let $A: C \rightarrow H$ be an $\alpha$-inverse-strongly monotone mapping and let $B$ be a maximal monotone operator on $H$. Let $f: C \rightarrow C$ be a contractive mapping with the constant $\beta \in[0,1)$. Assume that $\operatorname{Dom}(B) \subset C$ and $(A+B)^{-1}(0)$ is not empty. Let $J_{r_{n}}=(I+$ $\left.r_{n} B\right)^{-1}$ and let $\left\{x_{n}\right\}$ be a sequence generated in the following process: $x_{0} \in C$ and

$$
\left\{\begin{array}{l}
y_{n}=\alpha_{n} f\left(x_{n}\right)+\left(1-\alpha_{n}\right) x_{n}, \\
x_{n+1}=\beta_{n} x_{n}+\left(1-\beta_{n}\right) J_{r_{n}}\left(y_{n}-r_{n} A y_{n}+e_{n}\right), \quad \forall n \geq 0,
\end{array}\right.
$$

where $\left\{\alpha_{n}\right\}$ and $\left\{\beta_{n}\right\}$ are real number sequences in $(0,1)$ and $\left\{r_{n}\right\}$ is a positive real number sequence in $(0,2 \alpha)$. Assume that the control sequences satisfy the following restrictions:

(a) $\lim _{n \rightarrow \infty} \alpha_{n}=0, \sum_{n=0}^{\infty} \alpha_{n}=\infty$;

(b) $0<\liminf _{n \rightarrow \infty} \beta_{n} \leq \limsup _{n \rightarrow \infty} \beta_{n}<1$;

(c) $0<b \leq r_{n} \leq c<2 \alpha$ and $\sum_{n=1}^{\infty}\left|r_{n}-r_{n-1}\right|<\infty$;

(d) $\sum_{n=0}^{\infty}\left\|e_{n}\right\|<\infty$, 
where $b$ and c are two real numbers. Then $\left\{x_{n}\right\}$ converges strongly to a point $\bar{x} \in(A+B)^{-1}(0)$, where $\bar{x}=\operatorname{Proj}_{(A+B)^{-1}(0)} f(\bar{x})$.

Proof Put $S=I$, the identity on $H$. The desired conclusion can be obtained immediately.

Let $H$ be a Hilbert space and $f: H \rightarrow(-\infty,+\infty]$ a proper convex lower semicontinuous function. Then the subdifferential $\partial f$ of $f$ is defined as follows:

$$
\partial f(x)=\{y \in H: f(z) \geq f(x)+\langle z-x, y\rangle, z \in H\}, \quad \forall x \in H .
$$

From Rockafellar [16], we know that $\partial f$ is maximal monotone. It is easy to verify that $0 \in \partial f(x)$ if and only if $f(x)=\min _{y \in H} f(y)$. Let $I_{C}$ be the indicator function of $C$, i.e.,

$$
I_{C}(x)= \begin{cases}0, & x \in C, \\ +\infty, & x \notin C .\end{cases}
$$

Since $I_{C}$ is a proper lower semicontinuous convex function on $H$, we see that the subdifferential $\partial I_{C}$ of $I_{C}$ is a maximal monotone operator. Then $y=\left(I+\lambda \partial I_{C}\right)^{-1} x \Longleftrightarrow y=\operatorname{Proj}_{C} x$, $\forall x \in H, y \in C$.

Theorem 3.2 Let $A: C \rightarrow H$ be an $\alpha$-inverse-strongly monotone mapping. Let $S: C \rightarrow C$ be a strictly pseudocontractive mapping with the constant $\kappa \in[0,1)$ and let $f: C \rightarrow C$ be a contractive mapping with the constant $\beta \in[0,1)$. Assume that $F(S) \cap V I(C, A)$ is not empty. Let $\left\{x_{n}\right\}$ be a sequence generated in the following process: $x_{0} \in C$ and

$$
\left\{\begin{array}{l}
z_{n}=\alpha_{n} f\left(x_{n}\right)+\left(1-\alpha_{n}\right) x_{n}, \\
y_{n}=\operatorname{Proj}_{C}\left(z_{n}-r_{n} A z_{n}+e_{n}\right), \\
x_{n+1}=\beta_{n} x_{n}+\left(1-\beta_{n}\right)\left(\gamma_{n} y_{n}+\left(1-\gamma_{n}\right) S y_{n}\right), \quad \forall n \geq 0,
\end{array}\right.
$$

where $\left\{\alpha_{n}\right\},\left\{\beta_{n}\right\}$ and $\left\{\gamma_{n}\right\}$ are real number sequences in $(0,1)$ and $\left\{r_{n}\right\}$ is a positive real number sequence in $(0,2 \alpha)$. Assume that the control sequences satisfy the following restrictions:

(a) $\lim _{n \rightarrow \infty} \alpha_{n}=0, \sum_{n=0}^{\infty} \alpha_{n}=\infty$;

(b) $0<\liminf _{n \rightarrow \infty} \beta_{n} \leq \lim \sup _{n \rightarrow \infty} \beta_{n}<1$;

(c) $\kappa \leq \gamma_{n} \leq a<1, \lim _{n \rightarrow \infty}\left|\gamma_{n+1}-\gamma_{n}\right|=0$;

(d) $0<b \leq r_{n} \leq c<2 \alpha$ and $\sum_{n=1}^{\infty}\left|r_{n}-r_{n-1}\right|<\infty$;

(e) $\sum_{n=0}^{\infty}\left\|e_{n}\right\|<\infty$,

where $a, b$, and c are three real numbers. Then $\left\{x_{n}\right\}$ converges strongly to a point $\bar{x} \in F(S) \cap$ $(A+B)^{-1}(0)$, where $\bar{x}=\operatorname{Proj}_{F(S) \cap(A+B)^{-1}(0)} f(\bar{x})$.

Proof Put $B x=\partial I_{C}$. Next, we show that $V I(C, A)=\left(A+\partial I_{C}\right)^{-1}(0)$. Notice that

$$
\begin{aligned}
x \in\left(A+\partial I_{C}\right)^{-1}(0) & \Longleftrightarrow 0 \in A x+\partial I_{C} x \\
& \Longleftrightarrow-A x \in \partial I_{C} x \\
& \Longleftrightarrow\langle A x, y-x\rangle \geq 0 \\
& \Longleftrightarrow x \in V I(C, A) .
\end{aligned}
$$

We can conclude the desired conclusion immediately. 
If $S=I$, the identity on $H$, then we find from Theorem 3.1 the following result immediately.

Corollary 3.3 Let $A: C \rightarrow H$ be an $\alpha$-inverse-strongly monotone mapping. Let $S: C \rightarrow C$ be a strictly pseudocontractive mapping with the constant $\kappa \in[0,1)$ and let $f: C \rightarrow C$ be a contractive mapping with the constant $\beta \in[0,1)$. Assume that $F(S) \cap V I(C, A)$ is not empty. Let $\left\{x_{n}\right\}$ be a sequence generated in the following process: $x_{0} \in C$ and

$$
\left\{\begin{array}{l}
y_{n}=\alpha_{n} f\left(x_{n}\right)+\left(1-\alpha_{n}\right) x_{n}, \\
x_{n+1}=\beta_{n} x_{n}+\left(1-\beta_{n}\right) \operatorname{Proj}_{C}\left(y_{n}-r_{n} A y_{n}+e_{n}\right), \quad \forall n \geq 0
\end{array}\right.
$$

where $\left\{\alpha_{n}\right\}$ and $\left\{\beta_{n}\right\}$ are real number sequences in $(0,1)$ and $\left\{r_{n}\right\}$ is a positive real number sequence in $(0,2 \alpha)$. Assume that the control sequences satisfy the following restrictions:

(a) $\lim _{n \rightarrow \infty} \alpha_{n}=0, \sum_{n=0}^{\infty} \alpha_{n}=\infty$;

(b) $0<\liminf _{n \rightarrow \infty} \beta_{n} \leq \limsup _{n \rightarrow \infty} \beta_{n}<1$;

(c) $0<b \leq r_{n} \leq c<2 \alpha$ and $\sum_{n=1}^{\infty}\left|r_{n}-r_{n-1}\right|<\infty$;

(d) $\sum_{n=0}^{\infty}\left\|e_{n}\right\|<\infty$,

where $b$ and $c$ are three real numbers. Then $\left\{x_{n}\right\}$ converges strongly to a point $\bar{x} \in V I(C, A)$, where $\bar{x}=\operatorname{Proj}_{V I(C, A)} f(\bar{x})$.

Let $F$ be a bifunction of $C \times C$ into $\mathbb{R}$, where $\mathbb{R}$ denotes the set of real numbers. Recall the following equilibrium problem:

$$
\text { Find } x \in C \text { such that } F(x, y) \geq 0, \quad \forall y \in C \text {. }
$$

In this paper, we use $E P(F)$ to denote the solution set of the equilibrium problem (3.2).

To study the equilibrium problems (3.2), we may assume that $F$ satisfies the following conditions:

(A1) $F(x, x)=0$ for all $x \in C$;

(A2) $F$ is monotone, i.e., $F(x, y)+F(y, x) \leq 0$ for all $x, y \in C$;

(A3) for each $x, y, z \in C$,

$$
\limsup _{t \downarrow 0} F(t z+(1-t) x, y) \leq F(x, y)
$$

(A4) for each $x \in C, y \mapsto F(x, y)$ is convex and weakly lower semicontinuous.

Putting $F(x, y)=\langle A x, y-x\rangle$ for every $x, y \in C$, we see that the equilibrium problem (3.2) is reduced to a variational inequality.

Lemma 3.4 $[42,43]$ Let $C$ be a nonempty closed convex subset of $H$ and let $F: C \times C \rightarrow \mathbb{R}$ be a bifunction satisfying (A1)-(A4). Then, for any $r>0$ and $x \in H$, there exists $z \in C$ such that

$$
F(z, y)+\frac{1}{r}\langle y-z, z-x\rangle \geq 0, \quad \forall y \in C
$$

Further, define

$$
T_{r} x=\left\{z \in C: F(z, y)+\frac{1}{r}\langle y-z, z-x\rangle \geq 0, \forall y \in C\right\}
$$


for all $r>0$ and $x \in H$. Then, the following hold:

(a) $T_{r}$ is single-valued;

(b) $T_{r}$ is firmly nonexpansive, i.e., for any $x, y \in H$,

$$
\left\|T_{r} x-T_{r} y\right\|^{2} \leq\left\langle T_{r} x-T_{r} y, x-y\right\rangle
$$

(c) $F\left(T_{r}\right)=E P(F)$;

(d) $E P(F)$ is closed and convex.

Lemma 3.5 [44] Let $C$ be a nonempty closed convex subset of a real Hilbert space $H, F$ a bifunction from $C \times C$ to $\mathbb{R}$ which satisfies (A1)-(A4) and $A_{F}$ a multivalued mapping of $H$ into itself defined by

$$
A_{F} \mathcal{X}= \begin{cases}\{z \in H: F(x, y) \geq\langle y-x, z\rangle, \forall y \in C\}, & x \in C, \\ \emptyset, & x \notin C .\end{cases}
$$

Then $A_{F}$ is a maximal monotone operator with the domain $D\left(A_{F}\right) \subset C, E P(F)=A_{F}^{-1}(0)$ and

$$
T_{r} x=\left(I+r A_{F}\right)^{-1} x, \quad \forall x \in H, r>0,
$$

where $T_{r}$ is defined as in (3.3).

Theorem 3.6 Let $A: C \rightarrow H$ be an $\alpha$-inverse-strongly monotone mapping and let $F_{B}$ be a bifunction from $C \times C$ to $\mathbb{R}$ which satisfies (A1)-(A4). Let $S: C \rightarrow C$ be a strictly pseudocontractive mapping with the constant $\kappa \in[0,1)$ and let $f: C \rightarrow C$ be a contractive mapping with the constant $\beta \in[0,1)$. Assume that $F(S) \cap E P(F)$ is not empty. Let $\left\{x_{n}\right\}$ be a sequence generated in the following process: $x_{0} \in C$ and

$$
\left\{\begin{array}{l}
z_{n}=\alpha_{n} f\left(x_{n}\right)+\left(1-\alpha_{n}\right) x_{n}, \\
y_{n}=T_{r_{n}}\left(z_{n}-r_{n} A z_{n}+e_{n}\right), \\
x_{n+1}=\beta_{n} x_{n}+\left(1-\beta_{n}\right)\left(\gamma_{n} y_{n}+\left(1-\gamma_{n}\right) S y_{n}\right), \quad \forall n \geq 0,
\end{array}\right.
$$

where $\left\{\alpha_{n}\right\},\left\{\beta_{n}\right\}$, and $\left\{\gamma_{n}\right\}$ are real number sequences in $(0,1)$ and $\left\{r_{n}\right\}$ is a positive real number sequence in $(0,2 \alpha)$. Assume that the control sequences satisfy the following restrictions:

(a) $\lim _{n \rightarrow \infty} \alpha_{n}=0, \sum_{n=0}^{\infty} \alpha_{n}=\infty$;

(b) $0<\liminf _{n \rightarrow \infty} \beta_{n} \leq \limsup _{n \rightarrow \infty} \beta_{n}<1$;

(c) $\kappa \leq \gamma_{n} \leq a<1, \lim _{n \rightarrow \infty}\left|\gamma_{n+1}-\gamma_{n}\right|=0$;

(d) $0<b \leq r_{n} \leq c<2 \alpha$ and $\sum_{n=1}^{\infty}\left|r_{n}-r_{n-1}\right|<\infty$;

(e) $\sum_{n=0}^{\infty}\left\|e_{n}\right\|<\infty$,

where $a, b$, and c are three real numbers. Then $\left\{x_{n}\right\}$ converges strongly to a point $\bar{x} \in F(S) \cap$ $E P(F)$, where $\bar{x}=\operatorname{Proj}_{F(S) \cap E P(F)} f(\bar{x})$.

If $S=I$, the identity on $H$, then we find from Theorem 3.6 the following result on the equilibrium problem immediately. 
Corollary 3.7 Let $A: C \rightarrow H$ be an $\alpha$-inverse-strongly monotone mapping and let $F_{B}$ be a bifunction from $C \times C$ to $\mathbb{R}$ which satisfies (A1)-(A4). Let $f: C \rightarrow C$ be a contractive mapping with the constant $\beta \in[0,1)$. Assume that $E P(F)$ is not empty. Let $\left\{x_{n}\right\}$ be a sequence generated in the following process: $x_{0} \in C$ and

$$
\left\{\begin{array}{l}
y_{n}=\alpha_{n} f\left(x_{n}\right)+\left(1-\alpha_{n}\right) x_{n}, \\
x_{n+1}=\beta_{n} x_{n}+\left(1-\beta_{n}\right) T_{r_{n}}\left(y_{n}-r_{n} A y_{n}+e_{n}\right), \quad \forall n \geq 0,
\end{array}\right.
$$

where $\left\{\alpha_{n}\right\}$ and $\left\{\beta_{n}\right\}$ are real number sequences in $(0,1)$ and $\left\{r_{n}\right\}$ is a positive real number sequence in $(0,2 \alpha)$. Assume that the control sequences satisfy the following restrictions:

(a) $\lim _{n \rightarrow \infty} \alpha_{n}=0, \sum_{n=0}^{\infty} \alpha_{n}=\infty$;

(b) $0<\liminf _{n \rightarrow \infty} \beta_{n} \leq \limsup _{n \rightarrow \infty} \beta_{n}<1$;

(c) $0<b \leq r_{n} \leq c<2 \alpha$ and $\sum_{n=1}^{\infty}\left|r_{n}-r_{n-1}\right|<\infty$;

(d) $\sum_{n=0}^{\infty}\left\|e_{n}\right\|<\infty$,

where $b$ and $c$ are two real numbers. Then $\left\{x_{n}\right\}$ converges strongly to a point $\bar{x} \in E P(F)$, where $\bar{x}=\operatorname{Proj}_{E P(F)} f(\bar{x})$.

\section{Competing interests}

The authors declare that there is no competing interests.

\section{Authors' contributions}

The main idea of this paper was proposed by SYC. XQ and LW participate the research and performed some steps of the proof in this research. All authors read and approved the final manuscript.

\section{Author details}

'Department of Mathematics, Gyengsang National University, Jinju, 660-701, Korea. ²Department of Mathematics, Hangzhou Normal University, Hangzhou, 310036, China. ${ }^{3}$ Department of Mathematics, Faculty of Science, King Abdulaziz University, Jeddah, Saudi Arabia. ${ }^{4}$ College of Statistics and Mathematics, Yunnan University of Finance and Economics, Kunming, 650221, China.

\section{Acknowledgements}

The authors are grateful to the referees for useful suggestions which improved the contents of the paper.

Received: 5 January 2014 Accepted: 24 March 2014 Published: 09 Apr 2014

\section{References}

1. Browder, FE: Nonlinear operators and nonlinear equations of evolution in Banach spaces. Proc. Symp. Pure Math. 18, 78-81 (1976)

2. Browder, FE, Petryshyn, WV: Construction of fixed points of nonlinear mappings in Hilbert spaces. J. Math. Anal. Appl. 20, 197-228 (1967)

3. Cho, SY, Kang, SM: Approximation of common solutions of variational inequalities via strict pseudocontractions. Acta Math. Sci. 32, 1607-1618 (2011)

4. Cho, SY: Approximation of solutions of a generalized variational inequality problem based on iterative methods. Commun. Korean Math. Soc. 25, 207-214 (2010)

5. Cho, SY, Qin, X, Kang, SM: Iterative processes for common fixed points of two different families of mappings with applications. J. Glob. Optim. 57, 1429-1446 (2013)

6. Cho, SY, Kang, SM: Approximation of fixed points of pseudocontraction semigroups based on a viscosity iterative process. Appl. Math. Lett. 24, 224-228 (2011)

7. Luo, H, Wang, Y: Iterative approximation for the common solutions of a infinite variational inequality system for inverse-strongly accretive mappings. J. Math. Comput. Sci. 2, 1660-1670 (2012)

8. Cho, SY, Li, W, Kang, SM: Convergence analysis of an iterative algorithm for monotone operators. J. Inequal. Appl. 2013, Article ID 199 (2013)

9. Zegeye, $\mathrm{H}$, Shahzad, N: Strong convergence theorem for a common point of solution of variational inequality and fixed point problem. Adv. Fixed Point Theory 2, 374-397 (2012)

10. Rodjanadid, B, Sompong, S: A new iterative method for solving a system of generalized equilibrium problems, generalized mixed equilibrium problems and common fixed point problems in Hilbert spaces. Adv. Fixed Point Theory 3, 675-705 (2013)

11. Qin, X, Cho, SY, Kang, SM: An extragradient-type method for generalized equilibrium problems involving strictly pseudocontractive mappings. J. Glob. Optim. 49, 679-693 (2011)

12. Qin, $X$, Cho, SY, Kang, SM: Convergence of an iterative algorithm for systems of variational inequalities and nonexpansive mappings with applications. J. Comput. Appl. Math. 233, 231-240 (2009) 
13. Qin, X, Cho, SY, Kang, SM: Iterative algorithms for variational inequality and equilibrium problems with applications. J. Glob. Optim. 48, 423-445 (2010)

14. Browder, FE: Existence and approximation of solutions of nonlinear variational inequalities. Proc. Natl. Acad. Sci. USA 56, 1080-1086 (1966)

15. Rockafellar, RT: Augmented Lagrangians and applications of the proximal point algorithm in convex programming Math. Oper. Res. 1, 97-116 (1976)

16. Rockafellar, RT: Monotone operators and proximal point algorithm. SIAM J. Control Optim. 14, $877-898$ (1976)

17. Eckstein, J: Approximate iterations in Bregman-function-based proximal algorithms. Math. Program. 83, 113-123 (1998)

18. Khan, MA, Yannelis, NC: Equilibrium Theory in Infinite Dimensional Spaces. Springer, New York (1991)

19. Combettes, PL: The convex feasibility problem in image recovery. In: Hawkes, P (ed.) Advanced in Imaging and Electron Physcis, vol. 95, pp. 155-270. Academic Press, New York (1996)

20. Dautray, R, Lions, JL: Mathematical Analysis and Numerical Methods for Science and Technology, vol. 1. Springer, New York (1990)

21. Fattorini, HO: Infinite-Dimensional Optimization and Control Theory. Cambridge University Press, Cambridge (1999)

22. Güler, O: On the convergence of the proximal point algorithm for convex minimization. SIAM J. Control Optim. 29, 403-409 (1991)

23. Qin, X, Kang, SM, Cho, YJ: Approximating zeros of monotone operators by proximal point algorithms. J. Glob. Optim. 46, 75-87 (2010)

24. Song, J, Chen, M: A modified Mann iteration for zero points of accretive operators. Fixed Point Theory Appl. 2013, Article ID 347 (2013)

25. Qing, Y, Cho, SY: Proximal point algorithms for zero points of nonlinear operators. Fixed Point Theory Appl. 2014, Article ID 42 (2014)

26. Qing, Y, Cho, SY: A regularization algorithm for zero points of accretive operators. Fixed Point Theory Appl. 2013, Article ID 341 (2013)

27. Cho, SY, Kang, SM: Zero point theorems for $m$-accretive operators in a Banach space. Fixed Point Theory 13, 49-58 (2012)

28. Wu, C, Lv, S: Bregman projection methods for zeros of monotone operators. J. Fixed Point Theory 2013, Article ID 7 (2013)

29. Zhang, M: Iterative algorithms for common elements in fixed point sets and zero point sets with applications. Fixed Point Theory Appl. 2012, Article ID 21 (2012)

30. Qin, X, Su, Y: Approximation of a zero point of accretive operator in Banach spaces. J. Math. Anal. Appl. 329, 415-424 (2007)

31. Kim, JK, Anh, PN, Nam, YM: Strong convergence of an extended extragradient method for equilibrium problems and fixed point problems. J. Korean Math. Soc. 49, 187-200 (2012)

32. Qin, $X$, Cho, SY, Wang, L: Iterative algorithms with errors for zero points of $m$-accretive operators. Fixed Point Theory Appl. 2013, Article ID 148 (2013)

33. Solodov, MV, Svaiter, BF: Forcing strong convergence of proximal point iterations in a Hilbert space. Math. Program. 87, 189-202 (2000)

34. Zhang, M: Strong convergence of a viscosity iterative algorithm in Hilbert spaces. J. Nonlinear Funct. Anal. 2014 Article ID 1 (2014)

35. Qin, $X$, Shang, M, Su, Y: Strong convergence of a general iterative algorithm for equilibrium problems and variational inequality problems. Math. Comput. Model. 48, 1033-1046 (2008)

36. Cho, SY: Strong convergence of an iterative algorithm for sums of two monotone operators. J. Fixed Point Theory 2013, Article ID 6 (2013)

37. Barbu, V: Nonlinear Semigroups and Differential Equations in Banach Space. Noordhoff, Groningen (1976)

38. Suzuki, T: Strong convergence of Krasnoselskii and Mann's type sequences for one-parameter nonexpansive semigroups without Bochner integrals. J. Math. Anal. Appl. 305, 227-239 (2005)

39. Xue, Z, Zhou, H, Cho, YJ: Iterative solutions of nonlinear equations for $m$-accretive operators in Banach spaces. J. Nonlinear Convex Anal. 1, 313-320 (2000)

40. Zhou, $\mathrm{H}$ : Convergence theorems of fixed points for $\kappa$-strict pseudo-contractions in Hilbert spaces. Nonlinear Anal. 69, 456-462 (2008)

41. Lions, PL, Mercier, B: Splitting algorithms for the sum of two nonlinear operators. SIAM J. Numer. Anal. 16, 964-979 (1979)

42. Blum, E, Oettli, W: From optimization and variational inequalities to equilibrium problems. Math. Stud. 63, 123-145 (1994)

43. Fan, K: A minimax inequality and applications. In: Shisha, O (ed.) Inequality, vol. III, pp. 103-113. Academic Press, New York (1972)

44. Takahashi, S, Takahashi, W, Toyoda, M: Strong convergence theorems for maximal monotone operators with nonlinear mappings in Hilbert spaces. J. Optim. Theory Appl. 147, 27-41 (2010)

10.1186/1687-1812-2014-94

Cite this article as: Cho et al.: Strong convergence of a splitting algorithm for treating monotone operators. Fixed Point Theory and Applications 2014, 2014:94 